\title{
Finite-Size Dark Matter and its Effect on Small-Scale Structure
}

\author{
Xiaoyong Chu $\odot{ }^{1, *}$ Camilo Garcia-Cely, ${ }^{2, \dagger}$ and Hitoshi Murayama ${ }^{3,4,5,2, \$}$ \\ ${ }^{1}$ Institute of High Energy Physics, Austrian Academy of Sciences, Nikolsdorfer Gasse 18, 1050 Vienna, Austria \\ ${ }^{2}$ Deutsches Elektronen-Synchrotron DESY, Notkestrasse 85, 22607 Hamburg, Germany \\ ${ }^{3}$ Department of Physics, University of California, Berkeley, California 94720, USA \\ ${ }^{4}$ Kavli Institute for the Physics and Mathematics of the Universe (WPI), University of Tokyo, Kashiwa 277-8583, Japan \\ ${ }^{5}$ Ernest Orlando Lawrence Berkeley National Laboratory, Berkeley, California 94720, USA
}

(Received 3 February 2019; revised manuscript received 25 September 2019; published 28 January 2020)

\begin{abstract}
If dark matter has a finite size that is larger than its Compton wavelength, the corresponding selfinteraction cross section decreases with the velocity. We investigate the implications of this puffy dark matter for addressing the small-scale problems of the $\Lambda$ cold dark matter model and show that the way the nonrelativistic cross section varies with the velocity is largely independent of the dark matter internal structure. Even in the presence of a light particle mediating self-interactions, we find that the finite-size effect may dominate the velocity dependence. We present an explicit example in the context of a QCD-like theory and discuss possible ways to differentiate puffy dark matter from the usual light-mediator scenarios. Particularly relevant for this are low-threshold direct-detection experiments and indirect signatures associated with the internal structure of dark matter.
\end{abstract}

DOI: 10.1103/PhysRevLett.124.041101

Introduction.-Dark matter (DM) nature is one of the most important open questions of our century. Until now, we have only observed DM via its gravitational effects, with data supporting the hypothesis that DM is collisionless at large scales [1]. This is at the core of the celebrated $\Lambda$ cold dark matter $(\Lambda \mathrm{CDM})$ model, and its most stringent tests come from large objects such as clusters of galaxies, which constrain the self-scattering cross section per unit mass $\sigma / m$ to be below $1 \mathrm{~cm}^{2} / \mathrm{g}[2,3]$. Despite this, larger values of $\sigma / \mathrm{m}$ are not ruled out in small objects such as galaxies and dwarf spheroidals. Nonetheless, that requires the self-scattering cross section to decrease with the DM velocity, because the particles residing in larger DM halos move faster. This paradigm is known as self-interacting dark matter (SIDM) [4] and has attracted a lot of attention from astronomers and particle physicists in the last two decades.

One reason for this is the apparent mass deficit in the inner regions of small-scale halos with respect to the predictions of collisionless DM. This has led to the socalled small-scale crisis of the $\Lambda$ CDM model, which might be solved by SIDM because it predicts DM halos with smaller central densities [5-7] (for a review, see [8,9]). Another reason for the continued interest in SIDM is that it

Published by the American Physical Society under the terms of the Creative Commons Attribution 4.0 International license. Further distribution of this work must maintain attribution to the author(s) and the published article's title, journal citation, and DOI. Funded by SCOAP. gives clues about specific properties of DM, which can be used to search for it. For instance, large and velocitydependent cross sections might hint at a long-range force, which in turn suggests the presence of a light mediator. In fact, since such a particle is a rather generic feature of several well-motivated DM models, velocity-dependent SIDM is often associated with a light mediator. In this Letter, we discuss another source of velocity dependence for $\sigma / m$ (see also [10-15]), which hints at DM particles of finite size, $r_{\mathrm{DM}}$.

As is shown in Fig. 1, a momentum transfer much smaller than $r_{\mathrm{DM}}^{-1}$ is too small to measure the internal structure of the DM, so the latter acts as a pointlike particle. On the other hand, when the momentum transfer becomes larger than $r_{\mathrm{DM}}^{-1}$, the internal structure of the particle is probed. As specified below, this can happen in such a way that the phase difference among the scattered waves leads to a suppression in $\sigma / \mathrm{m}$. This is indeed the desired velocity dependence. In fact, as we will see, even in the presence of light mediators, the finite size may be the dominant effect.

We will refer to this scenario as puffy DM. Besides the self-scattering effects, the fact that DM has a finite size leads to a very rich phenomenology, as has been explored for several concrete DM candidates (e.g., [16-37]).

Scattering of finite-size DM particles.-Let us first consider the scattering of two finite-size objects, which -for simplicity-will be modeled as a collection of pointlike constituents that coherently scatter by means of a spin-independent Yukawa interaction. The corresponding charge density $\rho(\overrightarrow{\mathbf{r}})$ characterizes the finite shape of the scattering object. We will also assume that the contribution 


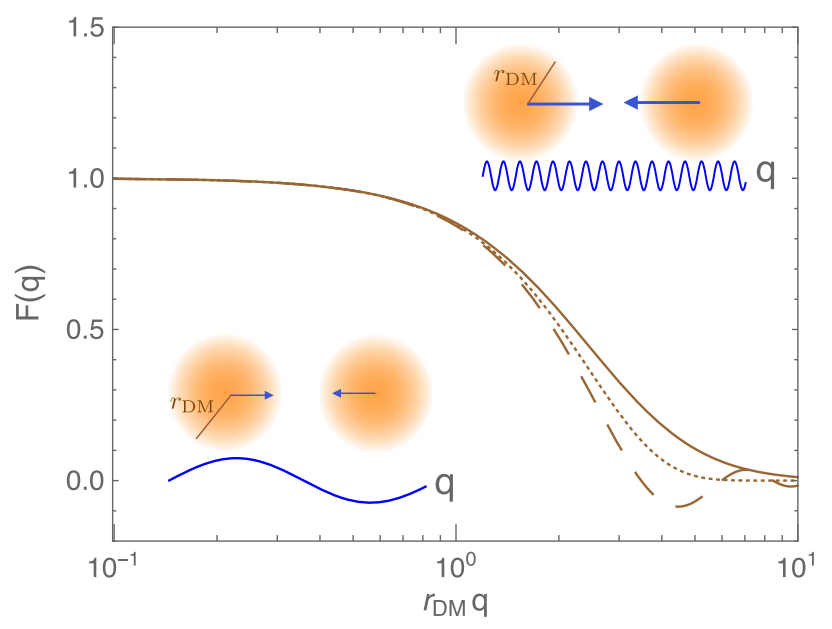

FIG. 1. Form factors as a function of momentum transfer $q$ in units of the inverse root-mean-square radius $r_{\mathrm{DM}}$. Solid, dashed, and dotted lines correspond to the dipole, top-hat, and Gaussian distributions (see Table I).

of the binding force to the scattering rate is negligible. This is the case, e.g., if such a force leads to a momentumsuppressed scattering amplitude. Then, the interaction Hamiltonian for two objects described by the density profiles $\rho_{1}(\overrightarrow{\mathbf{x}})$ and $\rho_{2}(\overrightarrow{\mathbf{y}})$ is

$$
\begin{aligned}
H_{\mathrm{int}} & =\int d \overrightarrow{\mathbf{x}} d \overrightarrow{\mathbf{y}} \rho_{1}(\overrightarrow{\mathbf{x}}) \frac{\alpha e^{-|\overrightarrow{\mathbf{x}}-\overrightarrow{\mathbf{y}}| / \lambda}}{|\overrightarrow{\mathbf{x}}-\overrightarrow{\mathbf{y}}|} \rho_{2}(\overrightarrow{\mathbf{y}}) \\
& =\int \frac{d \overrightarrow{\mathbf{q}}}{(2 \pi)^{3}} F_{1}(\overrightarrow{\mathbf{q}}) \frac{4 \pi \alpha}{\overrightarrow{\mathbf{q}}^{2}+\lambda^{-2}} F_{2}(-\overrightarrow{\mathbf{q}}),
\end{aligned}
$$

where $\lambda$ is the range of the interaction, $\alpha$ is a coupling constant, and we have introduced the form factor $F_{i}(\overrightarrow{\mathbf{q}}) \equiv \int d \overrightarrow{\mathbf{r}} e^{i \overrightarrow{\mathbf{q}} \cdot \overrightarrow{\mathbf{r}}} \rho_{i}(\overrightarrow{\mathbf{r}})$. Hence, the center-of-mass differential cross section in the Born approximation is

$$
\frac{d \sigma}{d \Omega}=S\left|F_{1}(\overrightarrow{\mathbf{q}}) \frac{2 \mu \alpha}{\overrightarrow{\mathbf{q}}^{2}+\lambda^{-2}} F_{2}(-\overrightarrow{\mathbf{q}}) \pm(\overrightarrow{\mathbf{q}} \rightarrow-\overrightarrow{\mathbf{q}})\right|^{2},
$$

where $\mu$ is the reduced mass and $\overrightarrow{\mathbf{q}}$ is the momentum transfer. For identical (nonidentical) particles, the second term must (not) be included and $S=1 / 2(1)$.

An illustrative example is the electron scattering off finite-size objects. This is determined by a Coulomb interaction $(\lambda \rightarrow \infty)$ with $\rho_{e}(\overrightarrow{\mathbf{r}})=\delta(\overrightarrow{\mathbf{r}})$ or $F_{e}(\overrightarrow{\mathbf{q}})=1$. In this case, Eq. (2) gives the well-known Rutherford scattering formula, which can be used to infer the shape of finitesize objects. When applied to the proton, one finds a density distribution decreasing exponentially with a characteristic scale $r_{0}^{-2}=0.71 \mathrm{GeV}^{2}$ [38]. The latter is the dipole distribution (see Table I), generally expected from wave function solutions to various potential wells [39].

We apply now Eq. (2) to nonrelativistic DM. Assuming that the DM particle is spherical, i.e., $F(\overrightarrow{\mathbf{q}})=F(q)$, the $S$-wave differential cross section reads

$$
\frac{d \sigma}{d \Omega}=\frac{\sigma_{0}}{8 \pi}\left(\frac{F(q)^{2}}{1+\lambda^{2} q^{2}}+(\theta \rightarrow \pi-\theta)\right)_{q=m v \sin \theta / 2}^{2},
$$

where $\sigma_{0}=4 \pi\left(m \alpha \lambda^{2}\right)^{2}$. Here $\theta$ and $v$ are, respectively, the scattering angle and the relative velocity in the center-ofmass frame. While the exact form of $\rho(r)$-and hence $F(q)$ in Eq. (3) — needs to be determined by solving for the wave function from the Schrödinger equation of the composite state, the differential cross section is not sensitive to the details of $\rho(r)$ as long as it is always positive (no screening) and it goes to zero sufficiently fast at large radii. In that case, the DM size-or more precisely-the root-meansquare radius

$$
r_{\mathrm{DM}}^{2} \equiv \int d \overrightarrow{\mathbf{r}} \rho(r) r^{2}=-\left.6 \frac{d^{2} F(q)}{d q^{2}}\right|_{q=0}
$$

is positive. Thus, $F(q)$ decreases for small momenta from $F(0)=\int d \overrightarrow{\mathbf{r}} \rho(r)$, which can be normalized to one without loss of generality. Figure 1 illustrates this for the three representative distributions as listed in Table I. Together with Eq. (3), all this implies that the cross section is constant at low velocities and eventually approaches zero, even if the range of the interaction is short.

DM scattering in astrophysical halos.-Because of the form factor, for low velocities we expect isotropic scattering, whereas for larger velocities forward scattering is more probable. Because of this, the transfer cross section $\sigma_{T} \equiv \int d \Omega(1-|\cos \theta|) d \sigma / d \Omega$ captures the self-interaction effects in DM halos better than $\sigma$ (see, e.g., [40]) and will be adopted below.

Figure 2 illustrates the dependence of $\sigma_{T}$ on the interaction range $\lambda$ and the particle size $r_{\mathrm{DM}}$. As apparent from the plot, $\sigma_{T}$ is largely independent of the exact expression for the form factor and therefore of $\rho(r)$.

TABLE I. Form factors for different density distributions.

\begin{tabular}{lccc}
\hline \hline Shape & $\rho(r)$ & $r_{\mathrm{DM}}$ & $F(q)$ \\
\hline Top hat & $\left(3 / 4 \pi r_{0}^{3}\right) \theta\left(r_{0}-r\right)$ & $2 \sqrt{3} r_{0}$ & $\left\{\left(3\left[\sin \left(r_{0} q\right)-r_{0} q \cos \left(r_{0} q\right)\right]\right) /\left(r_{0}^{3} q^{3}\right)\right\}$ \\
Dipole & {$\left[\left(e^{-r / r_{0}}\right) / 8 \pi r_{0}^{3}\right]$} & $\sqrt{3 / 5} r_{0}$ & $\left\{1 /\left[\left(1+r_{0}^{2} q^{2}\right)^{2}\right]\right\}$ \\
Gaussian & {$\left[1 /\left(8 r_{0}^{3} \pi^{3 / 2}\right)\right] e^{-r^{2} /\left(4 r_{0}^{2}\right)}$} & $\sqrt{6} r_{0}$ & $e^{-r_{0}^{2} q^{2}}$ \\
\hline \hline
\end{tabular}




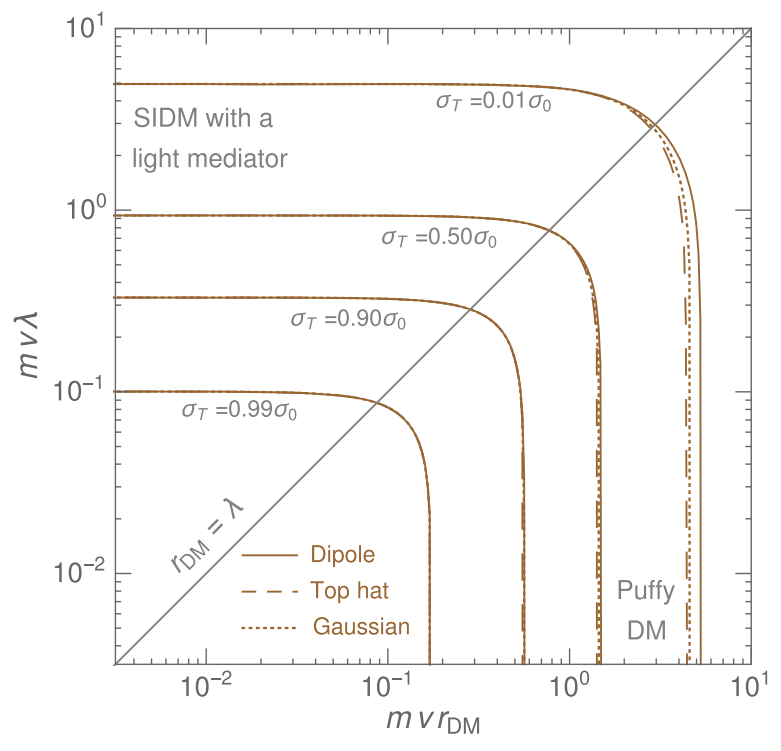

FIG. 2. Transfer cross section as a function of the force range $\lambda$ and the DM size $r_{\mathrm{DM}}$, both in units of $1 / m v$. Here $\sigma_{0}$ of Eq. (3) is assumed to be constant.

Furthermore, roughly speaking, the transfer cross section is constant for $m v \ll \min \left\{\lambda^{-1}, r_{\mathrm{DM}}^{-1}\right\}$, starts decreasing at $m v \sim \min \left\{\lambda^{-1}, r_{\mathrm{DM}}^{-1}\right\}$, and approximately scales as $1 / v^{4}$ for $m v \gg \min \left\{\lambda^{-1}, r_{\mathrm{DM}}^{-1}\right\}$ [41]. This directly follows from rewriting the transfer cross section as

$\sigma_{T}=\int_{0}^{\frac{(m v)^{2}}{2}} d q^{2}\left[\frac{F(q)^{2}}{1+\lambda^{2} q^{2}}+\left(q^{2} \rightarrow(m v)^{2}-q^{2}\right)\right]^{2} \frac{2 \sigma_{0} q^{2}}{(m v)^{4}}$.

When the range of the Yukawa force is much larger than the DM size, such a velocity dependence of $\sigma_{T}$ coincides with that of the Born regime of SIDM with a light mediator [11]. In fact, Fig. 2 shows that there is a one-to-one correspondence between the latter and the self-scattering of finite-size DM. Furthermore, there could be a mediator lighter than the DM and still the velocity dependence is determined by the DM size if $\lambda \lesssim r_{\mathrm{DM}}$.

The DM relative velocity in astrophysical halos typically follows a Maxwell-Boltzmann distribution truncated at the corresponding escape velocity $v_{\max }$. The velocity-averaged transfer cross section is then [42]

$$
\left\langle\sigma_{T} v\right\rangle=\int_{0}^{v_{\max }} f(v) \sigma_{T} v d v, \quad f(v)=\frac{32 v^{2} e^{-4 v^{2} / \pi\langle v\rangle^{2}}}{\pi^{2}\langle v\rangle^{3}} .
$$

In the context of SIDM as a solution to the small-scale structure problems, a semianalytical method has been proposed in [43] to infer, from observational data, the value of $\left\langle\sigma_{T} v\right\rangle / m$ for a given DM halo (see also [44]). This method was applied to five clusters from [45], seven lowsurface-brightness spiral galaxies in [46], and six dwarf galaxies of the HI Nearby Galaxy Survey sample [47]. Figure 3 shows these results, respectively, in green, blue, and red. The set of points is also in agreement with cluster bounds mentioned in the Introduction, giving $\sigma_{T} / m \lesssim$ $1.3 \mathrm{~cm}^{2} / \mathrm{g}[2,3]$. While cosmological simulations show this semianalytical method works for isolated halos (see, e.g., [48-50]), recent studies suggest that tidal stripping may further modify the density profile of satellite halos [51-54]. Such effects are not included here, because the galaxies shown in Fig. 3 are in the field.

Postulating a DM finite size much larger than the range of the Yukawa force, i.e., $\lambda \ll r_{\mathrm{DM}}$, provides an excellent fit to the velocity-dependent cross section preferred by the galactic and cluster systems. The corresponding best-fit of Eq. (6) to the data above is shown in Fig. 3 for the dipole, top-hat, and Gaussian distributions, separately. As expected from the aforementioned remarks, there is almost no dependence on details of the form factors, even though they correspond to substantially different density distributions. The figure also shows that, in order to have the right velocity dependence, the DM size needs to be hundreds of times larger than the Compton wavelength. This explains the name puffy DM.

If the Yukawa force is associated with a mediator $\rho$, requiring that the finite size dominates the scattering, i.e., $\lambda \lesssim r_{\mathrm{DM}}$, implies $m_{\rho} \gtrsim 10^{-3} \mathrm{~m}$. This shows that the mediator can still be substantially lighter than puffy DM. Moreover, if we impose $\alpha \lesssim m_{\rho} / m$ as required in the Born expansion, $\sigma_{0} / m=4 \pi\left(m \alpha \lambda^{2}\right)^{2} / m \sim 1 \mathrm{~cm}^{2} / \mathrm{g}$ leads to $m \lesssim 20 \mathrm{GeV}$. Consequently, puffy DM must lie at the $\mathrm{GeV}$ scale or below.

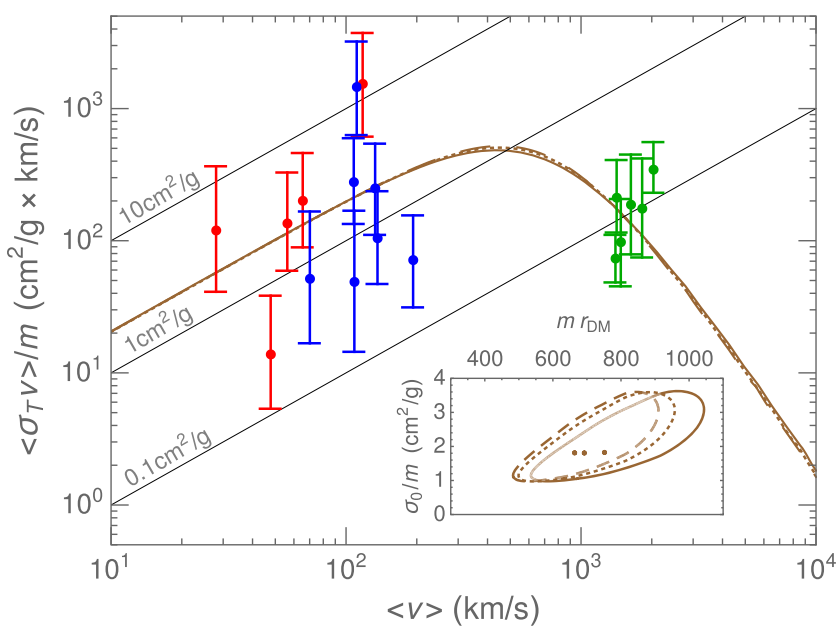

FIG. 3. Velocity dependence of the transfer cross section of puffy DM. Best-fit curves to data [43] for the dipole (solid), tophat (dashed), and Gaussian (dotted) distributions in Table I. (Inset) $95 \%$ C.L. contours on the parameter $\sigma_{0}$ from Eq. (3) and the DM size together with the corresponding parameter sets plotted in the main figure. 
A model of puffy DM.-Here we only sketch a possible realization of puffy DM; details will be discussed elsewhere. It is a QCD-like confining theory with $N_{c}$ colors and two flavors of quarks: one "charm quark," much heavier than the confining scale $\Lambda$, and one nearly massless "down quark." They, respectively, have charges $+2 / 3$ and $-1 / 3$ under a dark $\mathrm{U}(1)_{D}$ gauge group with $N_{c}=3$. This is associated with a massive "dark photon" $\gamma_{D}$, which can act as a portal to the Standard Model (SM) by means of the kinetic mixing between the $\mathrm{U}(1)_{D}$ group and the $\mathrm{SM}$ hypercharge. There are no dark weak interactions. We assume there is an asymmetry so that anticharm quarks are annihilated, while the remaining charm quarks end up in the baryonic $\Sigma_{c}(c d d)$ state. The latter interacts by exchanging the pseudoscalar $\eta(d \bar{d})$ and the vector $\rho(d \bar{d})$, which lead to attractive and repulsive forces, respectively.

On the one hand, it is likely that the $\eta$ exchange dominates binding $\Sigma_{c}$ baryons into nuclei because its range is larger given that the $\eta$ mass is due to the anomaly and hence suppressed as $m_{\eta} \sim \Lambda / \sqrt{N_{c}}$, as opposed to the $\rho$ mesons for which $m_{\rho} \sim \Lambda$. In view of this, in the following we assume the typical mass number is $10 \lesssim A \lesssim 100$. On the other hand, the nucleus-nucleus scattering is dominated by the exchange of $\rho$ mesons because the latter are essentially massive gauge bosons coupled to $d$ number $(A / 2)$ giving rise to coherent spin-independent scattering, while the $\eta$ exchange induces a spin-dependent momentum-suppressed scattering. Therefore, the range of the scattering force $\Lambda^{-1}$ is shorter than the size of the nuclei $r_{\mathrm{DM}} \sim A^{1 / 3} m_{\eta}^{-1} \sim A^{1 / 3} \Lambda^{-1} \sqrt{N_{c}}$. As a result, this model is a realization of puffy DM.

For instance, parameters such as $N_{c}=3, A \sim 10$, $m_{c} \sim m_{\Sigma_{c}} \sim 1 \mathrm{GeV}, \quad r_{\mathrm{DM}}^{-1} \sim 15 \mathrm{MeV}, \quad m_{\eta} \sim 20 \mathrm{MeV}$, $\Lambda \sim m_{\rho} \sim 30 \mathrm{MeV}$, and $\alpha \sim m_{\rho} / m$ realize the desired self-scattering cross section and its velocity dependence. We take $\gamma_{D}$ to be slightly lighter than $\eta$. Then the size of the kinetic mixing is either (i) $10^{-5} \lesssim \epsilon \lesssim 10^{-3}$ or (ii) $\epsilon \ll 10^{-10}$ to satisfy beam-dump experimental data and supernova observations $[36,55,56]$. In the cosmological history, presumably much of the entropy in this sector ends up in a thermally populated gas of $\eta$ mesons. These decay via $\eta \rightarrow \gamma_{D} \gamma_{D}^{*} \rightarrow 2\left(e^{+} e^{-}\right)$or self-annihilate via $\eta \eta \rightarrow \gamma_{D} \gamma_{D}$ before big bang nucleosynthesis for the range in (i) [57].

Direct-detection signatures are closely related to the DM finite size. The recoil-energy spectrum is that of a pointlike DM particle scattering via a contact interaction times the square of the dimensionless factor $\xi(q)=F_{D}(q) m_{\gamma_{D}}^{2} /$ $\left(q^{2}+m_{\gamma_{D}}^{2}\right)$. Its first part is the form factor associated with the $\mathrm{U}(1)_{D}$ charge and the second one parametrizes the dependence on the mediator mass. $F_{D}(0)=0$ because the $\mathrm{DM}$ particle is neutral under $\mathrm{U}(1)_{D}$ [58]. Thus, to leading order in $q, \xi(q) \simeq q^{2} F_{D}^{\prime \prime}(q=0) / 2$, which induces $\mathrm{DM}$ scattering rates enhanced by the fourth power of charge radius. The latter is given by Eq. (4) with $F \rightarrow F_{D}$ and is expected to be similar to $r_{\mathrm{DM}}^{4}$ on dimensional grounds. In view of this, we estimate current direct-detection limits by implementing such a recoil spectrum in DDCalc $[59,60]$. The results are shown in Fig. 4 for various choices of the charge radius and $m_{\gamma_{D}} \geq 20 \mathrm{MeV}$. For a lighter $\gamma_{D}$, the bound becomes more stringent than that due to an enhanced $\xi(q)$. Hence, Xenon1T significantly constrains DM masses above $5 \mathrm{GeV}$, while low-threshold direct-detection experiments such as CRESST-II and CDMSlite can probe smaller masses.

A salient aspect of this DM setup is that energy recoils are momentum suppressed albeit the enhancement due to the large charge radius. This is in sharp contrast to the direct detection of pointlike SIDM by means of light mediators. Since $q$ is proportional to the reduced mass of the colliding particles, we expect signals in experiments searching for nuclear recoils, but not in those looking for electron recoils, whose $\xi(q)$ is much smaller for the DM masses of interest here.

Likewise, the internal structure of puffy DM allows for up-scattering processes, giving rise to a wealth of indirect search signatures if DM de-excites ejecting SM particles. In our QCD-like model, this happens due to kinetic mixing, and for the parameters considered above, the required exciting energy can be estimated as $O\left(\Lambda^{2} / m_{c}\right) \sim \mathrm{MeV}$. While this is much greater than the typical galactic DM kinetic energy, DM might be excited by inelastic selfscatterings in massive clusters of galaxies or by collisions with high-energy cosmic rays [64-67]. The former case may lead to radio and x-ray signals (e.g., [68-70]) or DM dissipative cooling (e.g., [71-75]), while the latter might trigger novel signals in direct-detection and neutrino experiments as in, e.g., [76,77]. A detailed investigation is beyond the scope of this Letter.

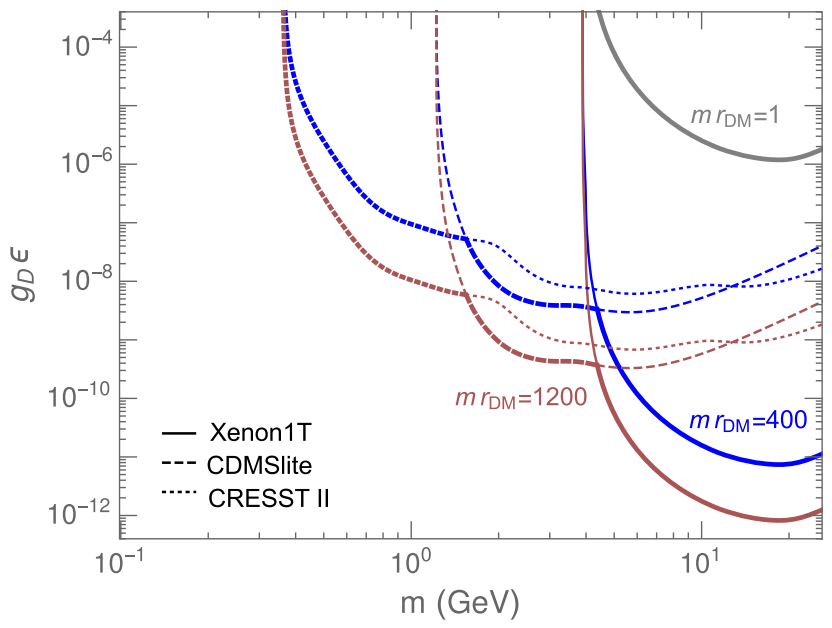

FIG. 4. Direct-detection bounds on our QCD-like theory of puffy DM from nucleon recoil events in Xenon1T [61], CMDSlite [62], and CRESST II [63], assuming $m_{\gamma_{D}}=$ $20 \mathrm{MeV}$. For a heavier dark photon, this bound scales with $m_{\gamma_{D}}^{2}$. See text for details. 
Before concluding, we would like to emphasize that puffy DM does not necessarily require QCD-like dynamics. Indeed, puffy DM can be realized in other theories of extended objects. For instance, (non-)topological defects, such as $Q$-balls [78-80] or skyrmions [81-83], are naturally stable, have a large size, and self-interact. The study of puffy DM in the form of defects is an ongoing project.

Conclusions.-We have shown that, if DM is an extended object with a size hundreds of times larger than its Compton wavelength, the corresponding self-interaction cross section varies with velocity in a way that is largely independent of its internal structure. For cross sections larger than $1 \mathrm{~cm}^{2} / \mathrm{g}$ at $v \rightarrow 0$, this provides a solution to the problems of the $\Lambda$ CDM model in small-scale astrophysical objects while still being in agreement with cluster observations. A QCD-like theory where DM is a dark nucleon has been used to illustrate our results, which are nevertheless general and can be applied to a broader range of theories. For this reason, we believe puffy DM opens up a new avenue for SIDM model building.

We thank Fady Bishara, Bob Cahn, Yohei Ema, Ranjan Laha, Kai Schmidt-Hoberg, and Sebastian Wild for enlightening discussions. X.C. is supported by the "New Frontiers" program of the Austrian Academy of Sciences. C. G. C. is supported by the ERC Starting Grant NewAve (638528). H. M. thanks the Alexander von Humboldt Foundation for support while this work was completed. H. M. was supported by the NSF Grant No. PHY-1638509, by the U.S. DOE Award No. DEAC02-05CH11231, by the JSPS Grant-in-Aid for Scientific Research (C) (17K05409), MEXT Grant-in-Aid for Scientific Research on Innovative Areas (15H05887, 15K21733), and by The World Premier International Research Center Initiative (WPI), MEXT, Japan.

*xiaoyong.chu@oeaw.ac.at

†camilo.garcia.cely@desy.de

†hitoshi@berkeley.edu, hitoshi.murayama@ipmu.jp

[1] N. Aghanim et al. (Planck Collaboration), Planck 2018 results. VI. Cosmological parameters, arXiv:1807.06209.

[2] S. W. Randall, M. Markevitch, D. Clowe, A. H. Gonzalez, and M. Bradac, Constraints on the self-interaction crosssection of dark matter from numerical simulations of the merging galaxy cluster 1E 0657-56, Astrophys. J. 679, 1173 (2008).

[3] A. Robertson, R. Massey, and V. Eke, What does the Bullet Cluster tell us about self-interacting dark matter?, Mon. Not. R. Astron. Soc. 465, 569 (2017).

[4] D. N. Spergel and P. J. Steinhardt, Observational Evidence for Self-Interacting Cold Dark Matter, Phys. Rev. Lett. 84, 3760 (2000).

[5] M. Vogelsberger, J. Zavala, and A. Loeb, Subhaloes in self-interacting galactic dark matter haloes, Mon. Not. R. Astron. Soc. 423, 3740 (2012).
[6] M. Rocha, A. H. G. Peter, J. S. Bullock, M. Kaplinghat, S. Garrison-Kimmel, J. Onorbe, and L. A. Moustakas, Cosmological simulations with self-interacting dark matter I: Constant density cores and substructure, Mon. Not. R. Astron. Soc. 430, 81 (2013).

[7] A. H. G. Peter, M. Rocha, J. S. Bullock, and M. Kaplinghat, Cosmological simulations with self-interacting dark matter II: Halo shapes vs. observations, Mon. Not. R. Astron. Soc. 430, 105 (2013).

[8] S. Tulin and H.-B. Yu, Dark matter self-interactions and small scale structure, Phys. Rep. 730, 1 (2018).

[9] J. S. Bullock and M. Boylan-Kolchin, Small-scale challenges to the $\Lambda \mathrm{CDM}$ paradigm, Annu. Rev. Astron. Astrophys. 55, 343 (2017).

[10] J. L. Feng, M. Kaplinghat, and H.-B. Yu, Halo Shape and Relic Density Exclusions of Sommerfeld-Enhanced Dark Matter Explanations of Cosmic Ray Excesses, Phys. Rev. Lett. 104, 151301 (2010).

[11] S. Tulin, H.-B. Yu, and K. M. Zurek, Beyond collisionless dark matter: Particle physics dynamics for dark matter halo structure, Phys. Rev. D 87, 115007 (2013).

[12] X. Chu, C. Garcia-Cely, and H. Murayama, Velocity Dependence from Resonant Self-Interacting Dark Matter, Phys. Rev. Lett. 122, 071103 (2019).

[13] S. D. McDermott, Is Self-Interacting Dark Matter Undergoing Dark Fusion?, Phys. Rev. Lett. 120, 221806 (2018).

[14] M. Vogelsberger, J. Zavala, K. Schutz, and T. R. Slatyer, Evaporating the Milky Way halo and its satellites with inelastic self-interacting dark matter, Mon. Not. R. Astron. Soc. 484, 5437 (2019).

[15] X. Chu and C. Garcia-Cely, Core formation from selfheating dark matter, J. Cosmol. Astropart. Phys. 07 (2018) 013.

[16] A. Coskuner, D. M. Grabowska, S. Knapen, and K. M. Zurek, Direct detection of bound states of asymmetric dark matter, Phys. Rev. D 100, 035025 (2019).

[17] S. Nussinov, Technocosmology: Could a technibaryon excess provide a 'natural' missing mass candidate?, Phys. Lett. B 165B, 55 (1985).

[18] R. S. Chivukula and T. P. Walker, Technicolor cosmology, Nucl. Phys. B329, 445 (1990).

[19] D. E. Kaplan, G. Z. Krnjaic, K. R. Rehermann, and C. M. Wells, Atomic dark matter, J. Cosmol. Astropart. Phys. 05 (2010) 021.

[20] B. Feldstein, A. L. Fitzpatrick, and E. Katz, Form factor dark matter, J. Cosmol. Astropart. Phys. 01 (2010) 020.

[21] K. Kumar, A. Menon, and T. M. P. Tait, Magnetic fluffy dark matter, J. High Energy Phys. 02 (2012) 131.

[22] R. Laha and E. Braaten, Direct detection of dark matter in universal bound states, Phys. Rev. D 89, 103510 (2014).

[23] J. M. Cline, Z. Liu, G. D. Moore, and W. Xue, Composite strongly interacting dark matter, Phys. Rev. D 90, 015023 (2014).

[24] M. B. Wise and Y. Zhang, Stable bound states of asymmetric dark matter, Phys. Rev. D 90, 055030 (2014); Erratum, Phys. Rev. D 91, 039907(E) (2015).

[25] G. Krnjaic and K. Sigurdson, Big bang darkleosynthesis, Phys. Lett. B 751, 464 (2015). 
[26] M. B. Wise and Y. Zhang, Yukawa bound states of a large number of fermions, J. High Energy Phys. 02 (2015) 023; Erratum, J. High Energy Phys. 10 (2015) 165(E).

[27] W. Detmold, M. McCullough, and A. Pochinsky, Dark nuclei I: Cosmology and indirect detection, Phys. Rev. D 90, 115013 (2014).

[28] E. Hardy, R. Lasenby, J. March-Russell, and S. M. West, Big bang synthesis of nuclear dark matter, J. High Energy Phys. 06 (2015) 011.

[29] E. Hardy, R. Lasenby, J. March-Russell, and S. M. West, Signatures of large composite dark matter states, J. High Energy Phys. 07 (2015) 133.

[30] R. Laha, Directional detection of dark matter in universal bound states, Phys. Rev. D 92, 083509 (2015).

[31] A. Mitridate, M. Redi, J. Smirnov, and A. Strumia, Dark matter as a weakly coupled dark baryon, J. High Energy Phys. 10 (2017) 210.

[32] M. I. Gresham, H. K. Lou, and K. M. Zurek, Astrophysical signatures of asymmetric dark matter bound states, Phys. Rev. D 98, 096001 (2018).

[33] A. Francis, R. J. Hudspith, R. Lewis, and S. Tulin, Dark matter from strong dynamics: The minimal theory of dark baryons, J. High Energy Phys. 12 (2018) 118.

[34] R. Contino, A. Mitridate, A. Podo, and M. Redi, Gluequark dark matter, J. High Energy Phys. 02 (2019) 187.

[35] E. Braaten, D. Kang, and R. Laha, Production of darkmatter bound states in the early universe by three-body recombination, J. High Energy Phys. 11 (2018) 084.

[36] M. Ibe, A. Kamada, S. Kobayashi, and W. Nakano, Composite asymmetric dark matter with a dark photon portal, J. High Energy Phys. 11 (2018) 203.

[37] M. Ibe, A. Kamada, S. Kobayashi, T. Kuwahara, and W. Nakano, Ultraviolet completion of a composite asymmetric dark matter model with a dark photon portal, J. High Energy Phys. 03 (2019) 173.

[38] C. F. Perdrisat, V. Punjabi, and M. Vanderhaeghen, Nucleon electromagnetic form factors, Prog. Part. Nucl. Phys. 59, 694 (2007).

[39] L. D. Landau and E. M. Lifshits, Quantum Mechanics, Vol. 3 of Course of Theoretical Physics (ButterworthHeinemann, Oxford, 1991).

[40] J. Kummer, F. Kahlhoefer, and K. Schmidt-Hoberg, Effective description of dark matter self-interactions in small dark matter haloes, Mon. Not. R. Astron. Soc. 474, 388 (2018).

[41] See Supplemental Material at http://link.aps.org/ supplemental/10.1103/PhysRevLett.124.041101 for more details.

[42] Here we will assume that $v_{\max } \rightarrow \infty$ since the integral converges quite fast due to the Boltzmann factor.

[43] M. Kaplinghat, S. Tulin, and H.-B. Yu, Dark Matter Halos as Particle Colliders: Unified Solution to Small-Scale Structure Puzzles from Dwarfs to Clusters, Phys. Rev. Lett. 116, 041302 (2016).

[44] M. Valli and H.-B. Yu, Dark matter self-interactions from the internal dynamics of dwarf spheroidals, Nat. Astron. 2, 907 (2018).

[45] A. B. Newman, T. Treu, R. S. Ellis, and D. J. Sand, The density profiles of massive, relaxed galaxy clusters: II. Separating luminous and dark matter in cluster cores, Astrophys. J. 765, 25 (2013).
[46] R. Kuzio de Naray, S. S. McGaugh, and W. J. G. de Blok, Mass models for low surface brightness galaxies with high resolution optical velocity fields, Astrophys. J. 676, 920 (2008).

[47] S.-H. Oh, W. J. G. de Blok, E. Brinks, F. Walter, and R. C. Kennicutt, Jr., Dark and luminous matter in THINGS dwarf galaxies, Astron. J. 141, 193 (2011).

[48] M. Vogelsberger, J. Zavala, F.-Y. Cyr-Racine, C. Pfrommer, T. Bringmann, and K. Sigurdson, ETHOS an effective theory of structure formation: Dark matter physics as a possible explanation of the small-scale CDM problems, Mon. Not. R. Astron. Soc. 460, 1399 (2016).

[49] P. Creasey, O. Sameie, L. V. Sales, H.-B. Yu, M. Vogelsberger, and J. Zavala, Spreading out and staying sharp creating diverse rotation curves via baryonic and selfinteraction effects, Mon. Not. R. Astron. Soc. 468, 2283 (2017).

[50] A. Sokolenko, K. Bondarenko, T. Brinckmann, J. Zavala, M. Vogelsberger, T. Bringmann, and A. Boyarsky, Towards an improved model of self-interacting dark matter haloes, J. Cosmol. Astropart. Phys. 12 (2018) 038.

[51] M. Kaplinghat, M. Valli, and H.-B. Yu, Too big to fail in light of Gaia, Mon. Not. R. Astron. Soc. 490, 231 (2019).

[52] O. Sameie, H.-B. Yu, L. V. Sales, M. Vogelsberger, and J. Zavala, Self-interacting dark matter subhalos in the Milky Way's tides, arXiv:1904.07872.

[53] J. Zavala, M. R. Lovell, M. Vogelsberger, and J. D. Burger, The diverse dark matter density at sub-kiloparsec scales in Milky Way satellites: Implications for the nature of dark matter, Phys. Rev. D 100, 063007 (2019).

[54] F. Kahlhoefer, M. Kaplinghat, T. R. Slatyer, and C.-L. Wu, Diversity in density profiles of self-interacting dark matter satellite halos, J. Cosmol. Astropart. Phys. 12 (2019) 010.

[55] E. Hardy and R. Lasenby, Stellar cooling bounds on new light particles: Plasma mixing effects, J. High Energy Phys. 02 (2017) 033.

[56] J. H. Chang, R. Essig, and S. D. McDermott, Revisiting supernova 1987A Constraints on dark photons, J. High Energy Phys. 01 (2017) 107.

[57] Assuming that $m_{\gamma_{D}} \leq m_{\eta}$ is not essential. If a dark axion $a$ exists, the annihilation $\eta \eta \rightarrow \eta a$ can play the same role.

[58] This is different from self-interaction form factor, where $F(0)=1$, while the charge radii of both should be of similar size.

[59] P. Athron et al. (GAMBIT Collaboration), Global analyses of Higgs portal singlet dark matter models using GAMBIT, Eur. Phys. J. C 79, 38 (2019).

[60] T. Bringmann et al. (The GAMBIT Dark Matter Workgroup Collaboration), DarkBit: A GAMBIT module for computing dark matter observables and likelihoods, Eur. Phys. J. C 77, 831 (2017).

[61] E. Aprile et al. (XENON Collaboration), First Dark Matter Search Results from the XENON1T Experiment, Phys. Rev. Lett. 119, 181301 (2017).

[62] R. Agnese et al. (SuperCDMS Collaboration), New Results from the Search for Low-Mass Weakly Interacting Massive Particles with the CDMS Low Ionization Threshold Experiment, Phys. Rev. Lett. 116, 071301 (2016). 
[63] G. Angloher et al. (CRESST Collaboration), Results on light dark matter particles with a low-threshold CRESST-II detector, Eur. Phys. J. C 76, 25 (2016).

[64] F. D'Eramo, K. Hambleton, S. Profumo, and T. Stefaniak, Dark matter inelastic up-scattering with the interstellar plasma: A new source of x-ray lines, including at 3.5 keV, Phys. Rev. D 93, 103011 (2016).

[65] C. V. Cappiello, K. C. Y. Ng, and J. F. Beacom, Reverse direct detection: Cosmic ray scattering with light dark matter, Phys. Rev. D 99, 063004 (2019).

[66] T. Bringmann and M. Pospelov, Novel Direct Detection Constraints on Light Dark Matter, Phys. Rev. Lett. 122, 171801 (2019).

[67] C. Cappiello and J. F. Beacom, Strong new limits on light dark matter from neutrino experiments, Phys. Rev. D 100, 103011 (2019).

[68] E. Storm, T. E. Jeltema, S. Profumo, and L. Rudnick, Constraints on dark matter annihilation in clusters of galaxies from diffuse radio emission, Astrophys. J. 768, 106 (2013).

[69] E. Storm, T. E. Jeltema, M. Splettstoesser, and S. Profumo, Synchrotron emission from dark matter annihilation: Predictions for constraints from non-detections of galaxy clusters with new radio surveys, Astrophys. J. 839, 33 (2017).

[70] P. Marchegiani, S. Colafrancesco, and N. F. Khanye, The role of dark matter annihilation in the radio emission of the galaxy cluster A520, Mon. Not. R. Astron. Soc. 483, 2795 (2019).

[71] J. Fan, A. Katz, L. Randall, and M. Reece, Double-disk dark matter, Phys. Dark Universe 2, 139 (2013).
[72] R. Foot, Galactic structure explained with dissipative mirror dark matter, Phys. Rev. D 88, 023520 (2013).

[73] J. Fan, A. Katz, and J. Shelton, Direct and indirect detection of dissipative dark matter, J. Cosmol. Astropart. Phys. 06 (2014) 059.

[74] K. K. Boddy, M. Kaplinghat, A. Kwa, and A. H. G. Peter, Hidden sector hydrogen as dark matter: Small-scale structure formation predictions and the importance of hyperfine interactions, Phys. Rev. D 94, 123017 (2016).

[75] R. Essig, H.-B. Yu, Y.-M. Zhong, and S. D. Mcdermott, Constraining Dissipative Dark Matter Self-Interactions, Phys. Rev. Lett. 123, 121102 (2019).

[76] G. F. Giudice, D. Kim, J.-C. Park, and S. Shin, Inelastic boosted dark matter at direct detection experiments, Phys. Lett. B 780, 543 (2018).

[77] Y. Ema, F. Sala, and R. Sato, Light Dark Matter at Neutrino Experiments, Phys. Rev. Lett. 122, 181802 (2019).

[78] A. Kusenko and M. E. Shaposhnikov, Supersymmetric Q balls as dark matter, Phys. Lett. B 418, 46 (1998).

[79] A. Kusenko, V. Kuzmin, M. E. Shaposhnikov, and P. G. Tinyakov, Experimental Signatures of Supersymmetric Dark Matter Q Balls, Phys. Rev. Lett. 80, 3185 (1998).

[80] A. Kusenko and P. J. Steinhardt, $Q$-Ball Candidates for SelfInteracting Dark Matter, Phys. Rev. Lett. 87, 141301 (2001).

[81] E. W. Mielke and F.E. Schunck, Nontopological scalar soliton as dark matter halo, Phys. Rev. D 66, 023503 (2002).

[82] M. Gillioz, A. von Manteuffel, P. Schwaller, and D. Wyler, The little skyrmion: New dark matter for little Higgs models, J. High Energy Phys. 03 (2011) 048.

[83] R. Kitano and M. Kurachi, Electroweak-skyrmion as topological dark matter, J. High Energy Phys. 07 (2016) 037. 